\title{
INTRODUCING ONLINE EXAMS
}

\author{
Jorge Borges 1, Elsa Justino 1, Carlos Vaz 1, João Barroso 1, 2 , Arsénio Reis 1, 2 \\ ${ }^{1}$ Universidade de Trás-os-Montes e Alto Douro (UTAD), Vila Real, PORTUGAL \\ ${ }^{2}$ Instituto de Engenharia de Sistemas e Computadores, Tecnologia e Ciência (INESC TEC), \\ Porto, PORTUGAL
}

\begin{abstract}
To receive a degree from a Higher Education Institution (HEI), the students must successfully undergo the necessary exams, which, most times, requires the physical presence of the student in a room where it will solve and answer the exams' questions using the traditional pen and paper. This is a wellknown and reliable method to evaluate the students, in which the physically presence is a reliable way to diminish the possibilities of fraud.

Currently, the modern Learning Management Systems (LMS) are able to provide the necessary features to execute the evaluations activities online, on the software platform. These systems and features can be use in a blended model, in which the students are required to be physically present, in a similar form to a traditional on site exam, and the exam is executed using a LMS. The exam room is equipped with Personal Computers (PC), which are configured with the necessary means to access the LMS and assure that a higher risk of fraud is not introduced by the usage of the PCs and the LMS as support for the exams. We designated this model, as the b-eval model.

The adoption of this model requires some changes in the professors' practices, as well as the introduction of more technological means to support the increase in PCs and LMS usage. The professors must design their evaluation process in order to incorporate the features and restrains posed by the PCs and the LMS. In some scenarios, this adoption is advantageous for the professor and for the students, considering that a properly designed exam can easily be executed by the students and instantaneously evaluated and graded. Otherwise, the traditional process takes much longer, as the professor must collect the paper exams, which he will later evaluate and grade. The process can take several days or even weeks.
\end{abstract}

In this position paper we discuss the introduction of the b-eval model, as well as an assessment methodology for its adoption.

Keywords: e-leaning, blended learning, higher education institution, learning management systems.

\section{INTRODUCTION}

Higher Education Institutions (HEls), usually, require the physical presence of their students for evaluation purposes. They haven't changed much, regarding the evaluation process, and continue to use a well-known and reliable method to evaluate their students at the end of the semester. The physically presence of the students is a traditional and reliable way to manage the possibilities of fraud.

The current modern Learning Management Systems (LMS) [1][2] can provide the necessary features to carry out online most of the evaluations activities, including the final semester exams. These systems and features can be use in a blended model, in which the students are required to be physically present, in a similar form to a traditional on site exam, and the exam is executed using a LMS through a connected device, e.g., a Personal Computer (PC) [3][4]. The exam room is equipped with PCs, which are equipped with the necessary means to access the LMS and assure that a higher risk of fraud is not introduced by the usage of PCs and LMS as support for the exams. We named this model, the b-eval model.

To adopt this model, professors must design their evaluation process in order to incorporate the features and restrains posed by the usage of the PCs and the LMS. In some scenarios, it is advantageous for the professor and for the students, as a proper designed exam can easily be executed by the students and instantaneously be evaluated and graded by the system. Otherwise, the traditional process takes much longer as the professor must collect the paper exams, which he will later evaluate, grade and publish the grades. The process can take up to several days or even weeks [5][6][7][8]. 
To change to the b-eval model the professor must consider the change in the specific practices required for the evaluation process, including: the exam design; the exam delivery to the students; and the exam evaluation and grading. A special care must be taken, regarding the accessibly issues, due to the introduction of technology [9][10].

In this position paper we discuss the adoption of the b-eval model and a methodology to assess the feasibility of introducing the on-line exams practices, by using the evaluation features of the LMS in a mode similar to the tradition exams [11].

\section{THE ASSESSMENT SCENARIOS}

We propose a simple assessment methodology that might be used by the large majority of the professors, to assess the pros and cons of the introduction of the b-eval model in their own courses and classes. The methodology should also provide a few metrics regarding the time and effort, used by the professors for the execution of the students' evaluation process.

In this methodology, we propose to model the evaluation process in three scenarios, corresponding to the oversimplification of the exams' design. These scenarios are related to current practices, commonly used in HEls.

\subsection{The scenarios}

We consider three types of exam design - the three scenarios, and for each scenario, it is proposed a 20 questions evaluation, with a mix composition of types of answers, including: direct answer, in which the student answer the question with a simple text phrase; multiple choice answer, requiring the student to choose an answer from a set of previously defined options; and open written answer, requiring the elaboration of an answer text.

For the purpose of this study, we consider the number of 20 questions, but in a real test, the 20 questions can be arranged in different groups, comprising questions and sub questions. We considered a total of 20 questions without regarding the test format or design structure.

\subsubsection{Scenario 1}

Of the three scenarios, the first is a test of 20 multiple choice questions. In this test, for each question, the student has a set of answers, from which he must choose the correct ones. There are some combinations of possible rules that can be used to regulate the student's choices, e.g., just one right answer, a declared number of right answers, etc. We don't detail this options on the proposal, and its usage should be up to the professors, considering the features of the LMS. The objective of this scenarios is to mimic and evaluate the exams designed according to the common types of multiple choice tests.

\subsubsection{Scenario 2}

In the second scenario, we propose an exam composition, in which the 20 questions are distributed in a mix of 8 multiple choice questions, plus 6 direct answers questions, and 6 written answer questions. This scenario should evaluate the introduction of minority part of the test as a multiple-choice test and a majority mix of direct answers and written answers. This scenario should represent the practices of the professors that use a mix of evaluation options on their exams' design.

\subsubsection{Scenario 3}

The third scenario proposes an exam design in which the professor uses, mainly, the tradition written answers. In this scenario. The exam is composed by a mix of 3 multiple choice questions plus 17 written answer questions.

\subsection{The assessment}

The assessment should be a comparison between the professor's current practices and the application of one of the scenarios. With the consistent application of this methodology, it should be created a reference regarding the three scenarios. That reference numbers would represent the expected time and effort necessary for a professor to execute his courses' evaluation, according to one of the stated scenarios. That way, the professor would only compare his current practices with the reference values, of effort and time, registered for the scenarios. 
At the institutional scale, it can be executed an assessment of benefits of the adoption of b-eval. We propose to analysed and estimate the number of courses or Curricular Units (UC) at the university that could be grouped in one of the three proposed scenarios, and estimated the benefits of keeping the actual evaluation model versus the adoption of the b-eval model. At a large scale, it should also be analysed the necessary technical and infrastructures means to support the b-eval model. A pure financial cost-benefit assessment should be executed, considering a five-year period, and distinct rates of equipment usage.

\section{THE INTRODUCTION OF THE B-EVAL MODEL}

The usage of the previously described methodology should provide a fast track for the adoption of the b-eval model. The introduction of on-line exams in this model should be assess individually, by each teacher and for each course. Besides the time and effort, previously assess, there are also other issues that should be taken into account.

The adoption of the b-eval model is dependent of the proper usage of the LMS by the professors, and although the LMS is not a user knowledge demanding application, it has some specific features that some professors may struggle with. A feasible approach to mitigate this problem, on an initial phase of adoption, is to deploy a proximity user support team to help the professors to design and create the online exams in the LMS.

By introducing the online exams, according to the b-eval model, we expect some indirect and unaccounted benefits, including:

1. the possibility of running analytics on the data store of a set of exams, providing indicators regarding student performance, teaching methodology effectiveness, professors' teaching success assessment;

2. the development of a massive and rich set of evaluation questions, regarding specific subjects, that can be used to simplify and automate the production of exams, including the creation of the exam within specific parameters or bias, such as, a determined difficulty degree.

The model also poses some general compliances issues regarding the current higher education regulation and country laws, namely, the necessity to comply with legal obligations, such as, the archive of the exams for a period of 5 after the exam's date. The digital support for the archive must include an infrastructure that has not considered in the current version of this study. Another issue that was not regarded is the user acceptance of the digital medium in contrast with the traditional paper medium. The study doesn't consider the students' perspective and focus mainly on the teaching practices. Also, regarding the students' perspective there should be a usability assessment, as the successful adoption of the model depends on usability issues well beyond the simple opinion or acceptance of the users, in this case, the students.

\section{CONCLUSIONS AND FUTURE WORK}

Using the assessment methodology we expect to provide a tool for the professors to easily assess a possible change in their current exams' practices. On another perspective, we expect to evaluate the impact (pros, cons, and gains) of the widely adoption of the b-eval model at the university.

In future work, a survey should be conducted in order to have a clear perspective of the current practices regarding the exams processes. Some aspects to consider should be: (1) the professors' effort and time, currently used in the exams' activities; (2) the classification of the types of exam's design used; (3) the motivation to adopt b-eval; (4) the required technological means.

\section{ACKNOWLEDGEMENTS}

This work was developed and financed by Project SAMA UNorteX.pt (Project number 022119, Code of Operation POCI-02-0550-FEDER-022119), Programa COMPETE 2020, Portugal 2020.

\section{REFERENCES}

[1] Vicent, L., and M. Segarra. "Learning management system." Multimedia in Education (2010): 2148. 
[2] Ifenthaler, Dirk. "Learning Management System." Encyclopedia of the Sciences of Learning. Springer US, 2012. 1925-1927.

[3] Valiathan, Purnima. "Blended learning models." Learning circuits 3.8 (2002): 50-59.

[4] Hillier, M. \& Fluck, A. "Arguing again for e-exams in high stakes examinations". Electric dreams. Proceedings of $30^{\text {th }}$ ascilite conference, 2013: 385-396.

[5] Luis Barbosa, Paulo Alves, Arsénio Reis, João Barroso: SIDE: Teaching Support Information System. Eunis 2011 Conference - Maintaining a Sustainable Future for IT in Higher Education, Dublin, Ireland; 01/2011.

[6] Harrington, C. F., \& Reasons, S. G. (2005). Online student evaluation of teaching for distance education: A perfect match. The Journal of Educators Online, 2(1), 1-12.

[7] Kuikka, M., Kitola, M. \& Laakso, M.J. "Challenges when introducing electronic exam". Research in Learning Technology, 2014. 22.

[8] Kuo, C.-Y. \&. Wu, H.-K. Toward an integrated model for designing assessment systems: An analysis of the current status of computer-based assessments in science. Computers \& Education, 2013. 68: 388-403.

[9] Arsénio Reis, João Barroso, Ramiro Gonçalves: Supporting Accessibility in Higher Education Information Systems. Proceedings of the 7th international conference on Universal Access in Human-Computer Interaction: applications and services for quality of life - Volume Part III; 07/2013, DOI:10.1007/978-3-642-39194-1_29.

[10] Terzis, V. \& Economides, A.A. The acceptance and use of computer based assessment. Computers \& Education, 2011. 56(4):1032-1044.

[11] Arsénio Monteiro Reis, Rio-Costa 15. A., Santos J., Borges J., Vasconcelos A., Barroso J., Bulas-Cruz J., Rodrigues F.: Providing Lifetime Services to Students. EUNIS International Conference 2009 "IT: Key of the European Space of Knowledge", Santiago de Compostela, Spain; 01/2009. 